\title{
Wladarsch, Jennifer: Metakommunikation und die Qualität des Journalismus. Einfluss von Metakommunikation auf Qualitätserwartungen und -bewertungen bei Nachrichtennutzern im Internet.
}

\author{
Baden-Baden: Nomos 2020. 339 Seiten. Preis: $€ 69$
}

\section{Annika Sehl}

Angenommen: 22. Dezember 2021 / Online publiziert: 11. Januar 2022

(C) Der/die Autor(en) 2022

In ihrer 2019 an der Ludwig-Maximilians-Universität München abgeschlossenen und von Christoph Neuberger betreuten Dissertation beschäftigt sich Jennifer Wladarsch mit der Frage, wie individuelle Qualitätsurteile über Onlinenachrichten entstehen. Dabei geht sie im Sinne der Sozialpsychologie von der Annahme aus, dass sich Nutzer:innen an den geäußerten Informationen, Bewertungen und dem Verhalten anderer Nutzer:innen orientieren, wenn sie journalistische Angebote im Internet rezipieren.

Mit ihrer Arbeit knüpft sie an den seit den frühen 1990er-Jahren in Deutschland bestehenden Forschungsstrang zur Qualität im Journalismus an, wählt jedoch gemäß des Erkenntnisinteresses einen anderen thematischen Zugang. Während frühere Studien vor allen die normative oder professionelle Sicht auf Qualität untersuchten, wählt Wladarsch die Publikumsperspektive, die erst in den vergangenen Jahren an Bedeutung gewann und noch viele Leerstellen aufweist.

Darüber hinaus unterscheidet sich ihre Arbeit von vielen anderen Arbeiten zur Qualität im Journalismus durch eine rein qualitative Vorgehensweise, statt auf standardisierte Erhebungsmethoden zu setzen. Somit können Nutzer:innenansprüche zwar nicht repräsentativ abgebildet, aber explorativ und jenseits vorher festgelegter Kriterien ergründet werden.

Der Theorieteil der Arbeit besteht auf drei Hauptkapiteln. Das erste befasst sich damit, wie journalistische Qualitätsurteile bei Nutzer:innen entstehen. Dabei folgt die Autorin Christoph Neuberger (2011) und definiert Qualität als subjektabhängige Wertung. Sie übernimmt dabei aus dem Wertungsmodell von Viktor Kraft (1951) die Komponenten Wertungssubjekt, Wertungsobjekt, Wert (Wertungsmaßstab) sowie

Prof. Dr. Annika Sehl $(\bowtie)$

Institut für Journalistik, Universität der Bundeswehr München,

Werner-Heisenberg-Weg 39, 85577 Neubiberg, Deutschland

E-Mail: annika.sehl@unibw.de 
Prädikat und ergänzt selbst eine fünfte Komponente, die Erwartung. Damit ist die voraussichtliche Wertung gemeint, noch bevor ein Wertungsobjekt rezipiert wird. Durch dieses Verständnis gelingt es ihr, die Urteilsgenese angemessen komplex zu diskutieren.

Das Qualitätsurteil versteht Wladarsch im Folgenden als die Differenz von Qualitätsansprüchen und Qualitätsbewertung individueller Rezipient:innen und bringt damit zusammen, was in anderen Studien oftmals getrennt behandelt wird. Die Entstehung von Qualitätsurteilen beschreibt sie prozessual und unterscheidet nach den Phasen der Mediennutzung (präkommunikativ, kommunikativ, postkommunikativ).

Im zweiten theoretischen Kapitel arbeitet die Autorin das Konzept der Metakommunikation als Einflussfaktor auf die Qualitätsbeurteilung im Journalismus aus. Dabei differenziert sie zwischen fremd- und angebotsseitiger Metakommunikation, was auch für folgende Arbeiten eine hilfreiche Einteilung sein kann. Fremdseitige Metakommunikation bezeichnet Fremdbewertungen oder -informationen (z. B. Nutzer:innenkommentare, Rezensionen). Angebotsseitige Metakommunikation dagegen umfasst Aussagen von Akteuren des Angebots (z. B. redaktionelle Empfehlungen) und Merkmale des Medienangebots selbst (z. B. Marken- oder Formatbezeichnungen). Fremdseitige wie auch angebotsseitige Metakommunikation können sowohl deskriptiv als auch evaluativ sein.

Das dritte theoretische Kapitel verknüpft die ersten beiden miteinander und stellt erste Forschungsergebnisse zum Einfluss der Metakommunikation auf die Genese von journalistischen Qualitätsurteilen bei Nutzer:innen dar. Wladarsch streift dabei verschiedenste theoretische Zugänge, sodass hier möglicherweise eine Konzentration auf weniger mehr gewesen wäre. Das Kapitel leitet dann zu den Forschungsfragen über. Die übergeordnete forschungsleitende Frage der Arbeit lautet: „Welchen Einfluss hat Metakommunikation auf die Genese von Qualitätsurteilen über professionell-journalistische Nachrichtenangebote im Internet bei Nutzern?“ (S. 167).

Aufbauend auf dem theoretischen Teil werden im Kapitel zu Forschungsdesign und Methodik Nutzer:innenkommentare, -empfehlungen und Beitragsrankings (als aggregierte und algorithmusbasierte Metainformationen) als konkrete Formen nutzer:innenseitiger Metakommunikation für die Untersuchung ausgewählt. Aus dem Nutzungsverhalten und der Qualitätsbewertung vielgenutzter Angebote wird zudem die Relevanz der Medienmarke sowie journalistischer Formen und Genre als angebotsseitige Metakommunikation abgeleitet.

Die Datenbasis der qualitativen Studie bilden 32 Leitfadeninterviews mit Nutzer:innen von Onlinenachrichten zwischen September und Dezember 2016. Sie wurden nach einem qualitativen Stichprobenplan ausgewählt, der die Intensität der Online-Nachrichtennutzung, das Alter und die formale Bildung umfasste.

Der im Vergleich zum Theorieteil sehr knappe Ergebnisteil zeigt - nicht überraschend und im Einklang mit bisheriger Forschung -, dass Medienmarken eine Signalwirkung sowohl auf Qualitätserwartungen als auch auf Qualitätsbewertungen haben. Auch Empfehlungen von anderen Nutzer:innen können Qualitätserwartungen beeinflussen, nämlich dann, wenn ihnen eine hohe Themen- oder Medienkompetenz zugeschrieben wird. In einer Typologie mit sechs Nutzer:innen-Typen beschreibt die Autorin unterschiedliche Arten und Stärken der Orientierung an anderen: „Beobach- 
ter“, „Unabhängige“, „Meinungsmacher“, „Informationssuchende“, „,Social-BubbleConsumer" und „Expertenkreis-Teilnehmer" (S. 274).

Die Arbeit zeichnet sich durch einen sehr umfassenden und weitgehend sorgfältig ausgearbeiteten Theorieteil, Systematik und vor allem bei der empirischen Untersuchung teils auch Kreativität aus. Gleichzeitig ist die Autorin selbst sehr reflektiert in Bezug auf die Limitationen ihrer Arbeit, u. a. dass Leitfadeninterviews nur geeignet sind, bewusste Prozesse bei den Nutzer:innen zu erfassen. Die größte Limitation, die aber für jede qualitative Studie gilt, ist, dass die geringe Anzahl an Leitfadeninterviews keine statistischen Zusammenhänge zulässt. Hier wäre es sehr wünschenswert, quantitativ und idealerweise mit einem repräsentativen Sample an diese Untersuchung anzuschließen. Denn beispielsweise Konstrukte wie Persönlichkeitstypen qualitativ und ohne systematische offene Fragen zu allen relevanten Aspekten zu erfassen, stellt die Ergebnisse teils auf tönerne Füße. Insbesondere die Typologie mit zum Teil nur ein oder zwei Befragten pro Nutzer:in-Typ bedarf einer größeren Datenbasis.

In Zeiten zunehmender Polarisierung könnten in Folgeuntersuchungen auch individuelle Einstellungen und Qualitätserwartungen sowie -bewertungen bei konkreten Themen, möglicherweise auch im Längsschnitt, erhoben werden, um die Entwicklung gesellschaftlicher (Konflikt-)diskurse einzubeziehen. In jedem Fall hat die theoretisch fundierte und methodisch akribische Arbeit von Jennifer Wladarsch den Qualitätsdiskurs mit dem Fokus auf Nutzer:innen und Qualitätserwartungen und -bewertungen im Internet um neue Einsichten bereichert, an die quantitativ angeknüpft werden sollte und die zusätzlich zu Anschlussfragen inspirieren.

Funding Open Access funding enabled and organized by Projekt DEAL.

Open Access Dieser Artikel wird unter der Creative Commons Namensnennung 4.0 International Lizenz veröffentlicht, welche die Nutzung, Vervielfältigung, Bearbeitung, Verbreitung und Wiedergabe in jeglichem Medium und Format erlaubt, sofern Sie den/die ursprünglichen Autor(en) und die Quelle ordnungsgemäß nennen, einen Link zur Creative Commons Lizenz beifügen und angeben, ob Änderungen vorgenommen wurden.

Die in diesem Artikel enthaltenen Bilder und sonstiges Drittmaterial unterliegen ebenfalls der genannten Creative Commons Lizenz, sofern sich aus der Abbildungslegende nichts anderes ergibt. Sofern das betreffende Material nicht unter der genannten Creative Commons Lizenz steht und die betreffende Handlung nicht nach gesetzlichen Vorschriften erlaubt ist, ist für die oben aufgeführten Weiterverwendungen des Materials die Einwilligung des jeweiligen Rechteinhabers einzuholen.

Weitere Details zur Lizenz entnehmen Sie bitte der Lizenzinformation auf http://creativecommons.org/ licenses/by/4.0/deed.de.

Prof. Dr. Annika Sehl ist Professorin für Digitalen Journalismus an der Universität der Bundeswehr München. 